Article

\title{
Cyclobutane-Containing Scaffolds as Useful Intermediates in the Stereoselective Synthesis of Suitable Candidates for Biomedical Purposes: Surfactants, Gelators and Metal Cation Ligands
}

\author{
Ona Illa *, Albert Serra, Agustí Ardiaca, Xavier Herrero, Guillem Closa and Rosa M. Ortuño * \\ Departament de Química, Universitat Autònoma de Barcelona, Cerdanyola del Vallès, 08193 Barcelona, Spain \\ * Correspondence: ona.illa@uab.es (O.I.); rosa.ortuno@uab.es (R.M.O.); Tel.: +34-935-811-602 (R.M.O.)
}

Received: 17 August 2019; Accepted: 2 September 2019; Published: 4 September 2019

check for updates

\begin{abstract}
Efficient and versatile synthetic methodologies are reported for the preparation of products that are suitable candidates to be used as surfactants, gelators for hydroxylic solvents or metal cation ligands, with potential use in several fields including biomedical applications. The common structural feature of all the synthesized products is the presence of a cis or trans-1,2- or cis-1,3-difunctionalized cyclobutane ring. In the two first cases, the key intermediates including enantiomerically pure 1,3-diamines and 1,3-amino alcohols have been prepared from $\beta$-amino acid derivatives obtained, in turn, from a chiral half-ester. This compound is also precursor of $\gamma$-amino esters. Furthermore, two kind of polydentate ligands have also been synthesized from a symmetric 1,5-diamine obtained from norpinic acid, which was easily prepared from commercial verbenone.
\end{abstract}

Keywords: cyclobutane; amphiphiles; surfactants; gelators; cation ligands

\section{Introduction}

Search for new molecules with suitable properties for biological purposes needs a rational synthetic design involving stereo- and chemoselective methodologies. Among target products, amphiphiles in general and, especially, surfactants and gelators play a key role in biomedicine. In addition, appropriate ligands to chelate cations are crucial for imaging technologies and radiopharmacy.

Amphiphiles are interesting and versatile compounds with usefulness in different fields. They can be classified in different families regarding their structure. Single head-single tail amphiphiles consist of an apolar tail and a polar head. Depending on the nature of the latter, they are classified as cationic, anionic or non-ionic amphiphiles [1]. They present a great potential for biomedical applications in targeted drug delivery systems (DDS) [1] and as non-viral vectors for gene therapy [2]. For the last purpose, cationic surfactants are especially interesting due to the fact of their properties that make them suitable to interact with DNA. Indeed, controlled DNA compaction and neutralization of their negatively charged phosphate groups is crucial to transfect into cells avoiding repulsive interactions with phospholipids in the cell membrane [3].

Otherwise, amphiphiles with properties as gelators and, in particular low-molecular-weight gelators (LMWG) are also valuable for the design and preparation of soft materials with utility in biochemical and biophysical studies [4] and applications in biomedicine, which include DDS, biosensors and tissue regeneration [5].

Imaging is nowadays a powerful tool in diagnosis and personalized medicine. In this area, contrast agents play a relevant role [6]. Many of them consist of appropriate metal complexes that must present specific requirements to assure their thermodynamic and kinetic stability to avoid metal leaching into the body. For this reason, the search for modifications in the structure of the ligands, 
trying to improve the properties and the effectiveness of the corresponding complexes, is the subject of a very active interdisciplinary research [7].

Previously, we have developed efficient synthetic methodologies to prepare both 1,2- and 1,3- disubstituted cyclobutane derivatives, whose exploitation in the synthesis of several kinds of amphiphiles [8-10], organogelators producing chiral aggregates [11,12] and metal ligands [13] has been explored. The presence of the cyclobutane ring confers all these types of molecules with rigidity as well as with two stereogenic centers, the relative and absolute configurations of which can be controlled. Scheme 1 summarizes synthetic strategies to prepare some cis- and trans-1,2-cyclobutane derivatives from a chiral half-ester that is the common precursor to cis- [14] and trans- $\beta$-amino acids $[15,16]$. From these compounds, we synthesized peptides with properties as foldamers in solution [16-18] and/or LMWG [19,20] as well as neuropeptide Y (NPY) analogues [21]. Amino alcohols [22] and diamines [22,23] have also been prepared, some of them with application in organocatalysis [22,23]. The 1,3-diamine motif is found in natural products and also in the structure of ligands for metal catalysts. Despite the many synthetic methods described to prepare 1,2-diamine building blocks, the synthetic approaches to 1,3-diamines are more limited [24-26].

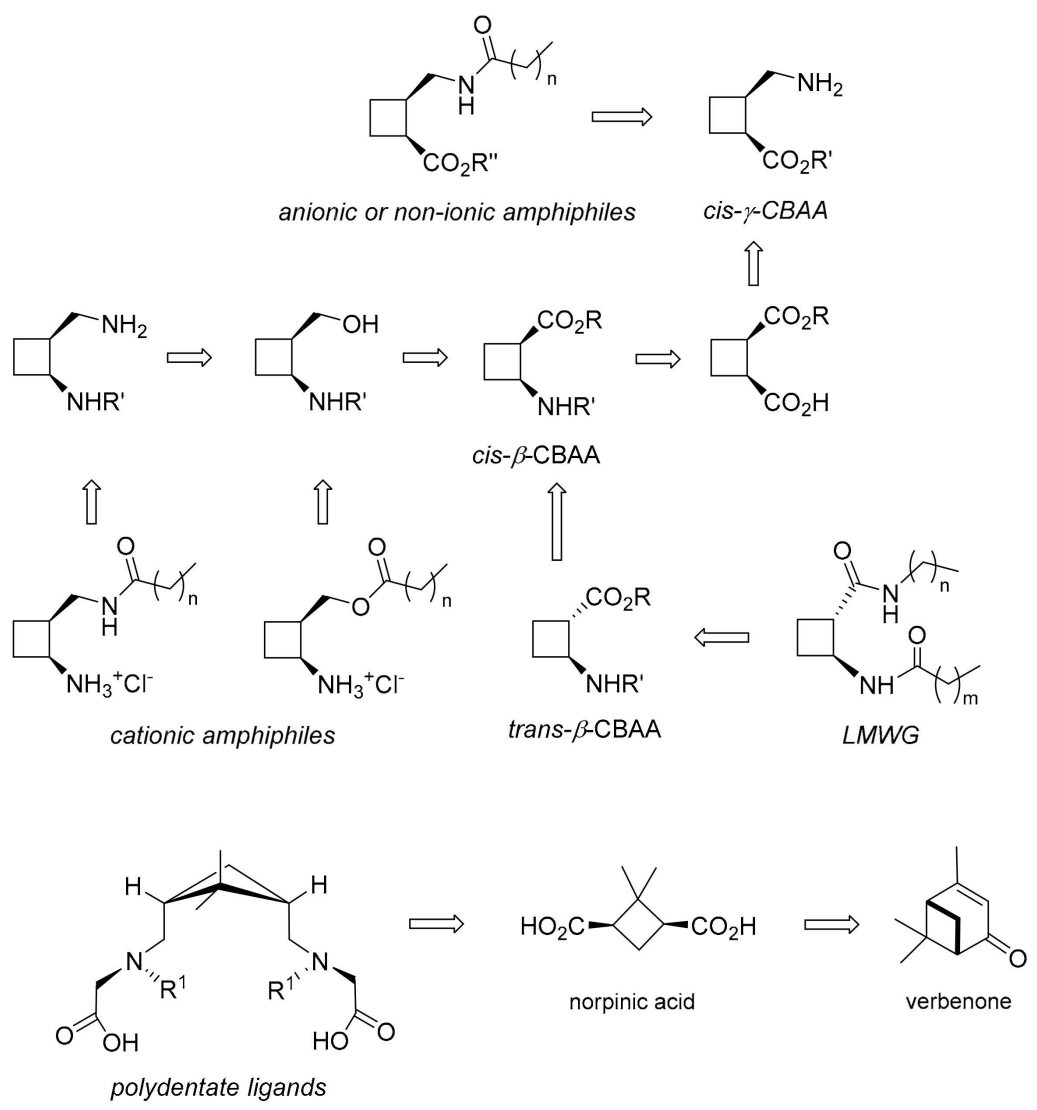

Scheme 1. Retrosynthetic strategies to prepare the target products from suitable precursors.

Concerning cis-1,3-disubstituted cyclobutane derivatives, we prepared organocatalysts [27] and other products as dendrimers [28] and cell penetrating peptides [29] starting from commercial verbenone, which provides polyfunctional chemical platforms that are highly useful scaffolds for synthetic applications.

In this article, we describe the utilization of cis- and trans-cyclobutane $\beta$-amino acids ( $\beta$-CBAA) [30] in the stereoselective synthesis of novel chiral cationic surfactants and LMWG. Moreover, a new synthesis of $c i s-\gamma$-CBAA derivatives is provided along with their application as synthetic precursors to anionic or non-ionic amphiphiles. Some examples are described herein to illustrate the usefulness of these methodologies (Scheme 1). 
Moreover, highly rigid polydentate ligands for metal cations were synthesized as well from norpinic acid [31], which is easily obtained from verbenone (Scheme 1) or pinene. Related ligands, previously described in the literature, have formed gadolinium complexes with interesting properties as potential candidates to contrast agents for magnetic resonance imaging [32-34], or complexes with other cations of application in radiopharmacy [35] or for industrial purposes [36].

\section{Results and Discussion}

\subsection{Amphiphiles from 1,2-Disubstituted Cyclobutane Scaffolds: Surfactants and LMWG}

Scheme 1 shows two retrosynthetic strategies to prepare different families of single head-single tail amphiphiles. Cationic derivatives were synthesized from 1,3-diamines and 1,3-amino alcohols, respectively, as the immediate precursors. These compounds, in turn, were obtained from orthogonally protected cis- $\beta$-CBAA through selective transformations of the functional groups. A second synthetic route leads to the formation of $c i s-\gamma$-CBAA as the key intermediates, which allows the preparation of anionic or non-ionic amphiphiles. Moreover, trans- $\beta$-CBAA, obtained through regioselective epimerization of the cis diastereomers, led to molecules which had structural features that conferred on them possible properties as organogelators (Scheme 1). Indeed, these compounds bear functional groups suitable for intermolecular hydrogen bonding and appropriate long alkyl-chains for van der Waals interactions. In addition, trans stereochemistry precludes the formation of intramolecular hydrogen bonds, thus favouring the interactions with solvents.

The synthetic route towards non-ionic or anionic amphiphiles is depicted in Scheme 2. The carboxyl group in half-ester $\mathbf{1}$ [14] was selectively reduced with diborane in tetrahydrofuran, at $0{ }^{\circ} \mathrm{C}$, providing primary alcohol 2 in $63 \%$ yield, which was activated towards nucleophilic displacement to introduce the amino group. Thus, treatment of $\mathbf{2}$ with tosyl chloride in the presence of triethylamine (TEA), giving tosylate 3 in $77 \%$ yield, and subsequent reaction with sodium azide followed by hydrogenation in the presence of $20 \% \mathrm{Pd}(\mathrm{OH})_{2}$ on activated charcoal as a catalyst provided amino ester 4 . Reaction of this intermediate with lauryl chloride led to 5 in $51 \%$ yield ( 3 steps), which is representative of non-ionic amphiphiles. Moreover, deprotection of the carboxyl group, by acid cleavage of the tert-butyl ester with trifluoroacetic acid and triethylsilane in dichloromethane, afforded quantitatively 6 , which would be the direct precursor of an anionic amphiphile via deprotonation of the carboxylic acid.

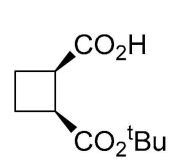

1
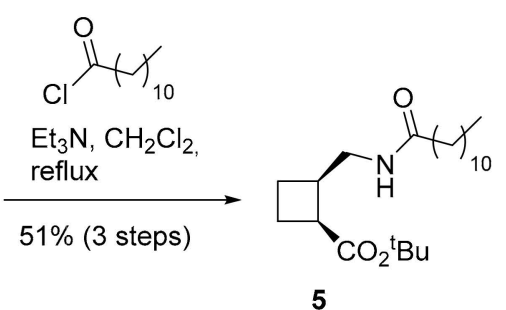

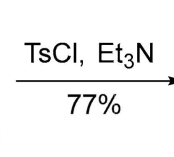

2

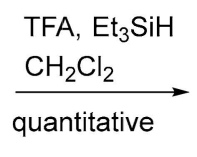

quantitative
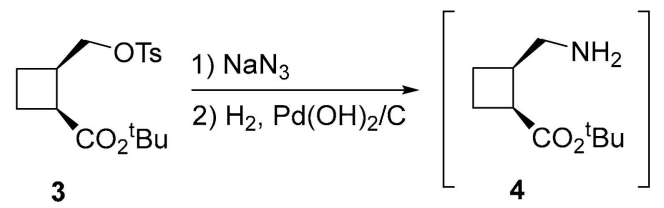

3

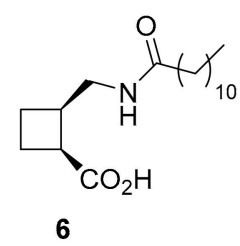

Scheme 2. Synthetic route towards non-ionic or anionic amphiphiles.

Scheme 3 shows the synthetic pathways to prepare cationic amphiphiles $\mathbf{1 2}$ and $\mathbf{1 4}$ and LMWG $\mathbf{1 8}$ and 19 from cis- and trans-CBAA, respectively. The methyl ester in precursor 7 [14] was reduced with lithium borohydride in ether to afford alcohol 8 in $75 \%$ yield. This compound was submitted to similar transformations as those described above, giving diamine $\mathbf{1 0 .}$ 


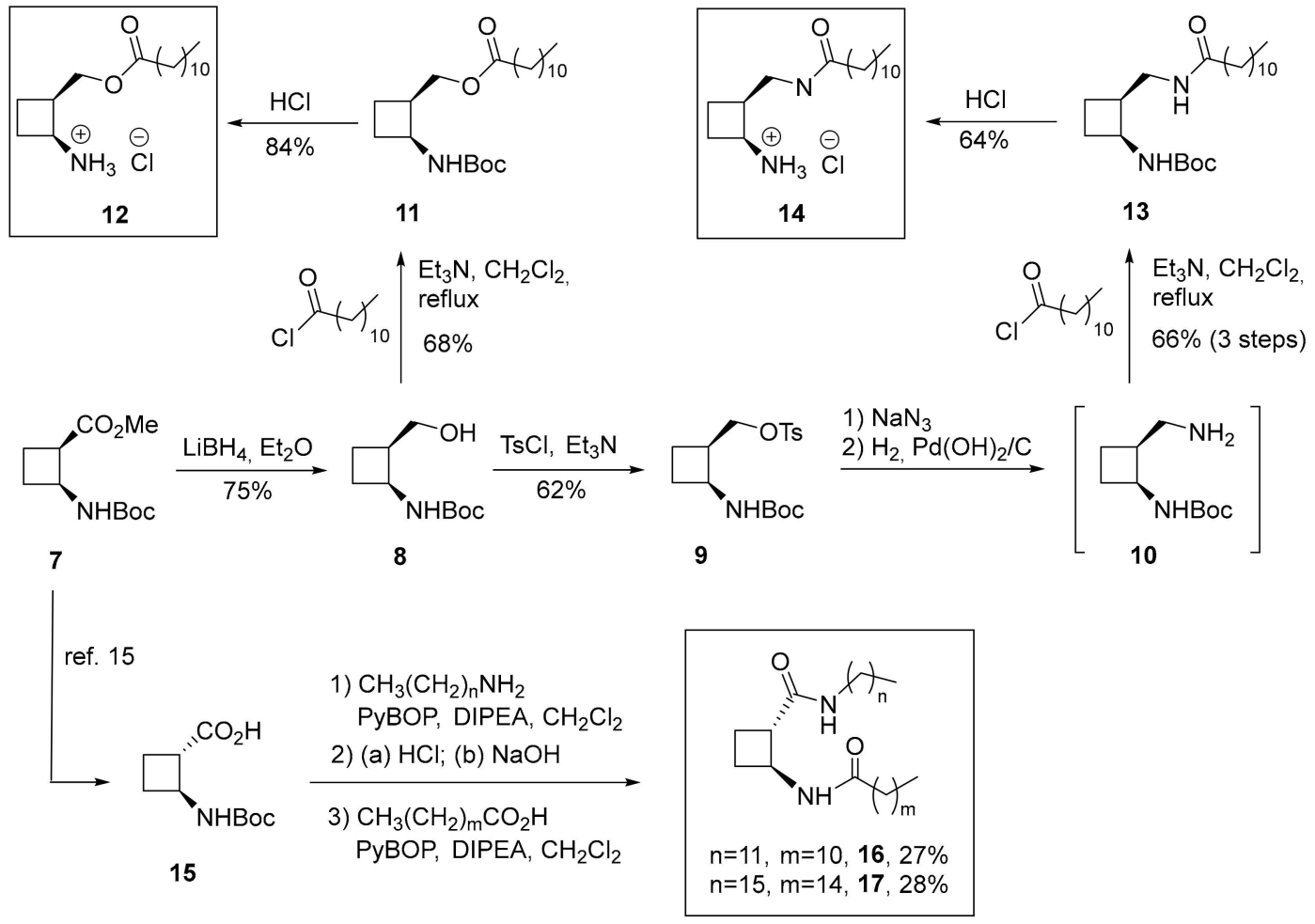

Scheme 3. Synthesis of cationic amphiphiles and organogelators from cis- or trans- $\beta$-CBAA.

Separate reactions of amino alcohol $\mathbf{8}$ and diamine $\mathbf{1 0}$ with lauryl chloride and TEA under reflux of dichloromethane led to compounds 11 and 13, respectively, in 66-68\% yield. Treatment of these products with $2 \mathrm{~N} \mathrm{HCl}$ in dichloromethane allowed to obtain cationic surfactants $\mathbf{1 2}$ and $\mathbf{1 4}$ in $84 \%$ and $64 \%$, respectively.

The synthetic route for the preparation of LMWG 16 and 17 (Scheme 3) starts with coupling of previously described trans- $\beta$-CBAA 15 [15] with dodecyl- or hexadecylamine, using (benzotriazol-1yloxy)tripyrrolidinophosphonium hexafluorophosphate (РyBOP) as a coupling agent in the presence of diisopropylethylamine (DIPEA) in dichloromethane, at room temperature, afforded the corresponding amides that, in separate processes, were submitted to amine deprotection by treatment with $\mathrm{HCl}$ followed of aqueous $\mathrm{NaOH}$ to provide the free amines. Further coupling with lauric or palmitic acid, under similar conditions as before, led to diamides 16 and 17, respectively, in $27-28 \%$ yield from 15 . Preliminary experiments showed that these compounds are good gelators for alcohols $(\mathrm{MeOH}, \mathrm{EtOH}, \mathrm{i}-\mathrm{PrOH})$ with minimum gelation concentration $(m g c)$ values $8-47 \mathrm{mg} / \mathrm{mL}$ and biocompatible $\mathrm{EtOH}$-water mixtures (up to $30 \%$ water) with $m g c=10-16 \mathrm{mg} / \mathrm{mL}$. These data differentiate these LMWG from alkyl bisamides prepared from cyclobutane-1,2-dicarboxylic acid, which were insoluble in alcohols [11], and confer these products with very interesting features for future applications.

\subsection{Highly Rigid Polydentate Ligands From 1,3-disubstituted Cyclobutane Scaffolds}

1,5-Diamine 19 was synthesized from previously known dimesylate 18 (Scheme 4), which had been obtained from norpinic acid [31]. The procedure was similar to that described above for the preparation of amines $\mathbf{4}$ and $\mathbf{1 0 .}$

Tetraalkylation of diamine 19 with tert-butyl bromoacetate, in the presence of KI and DIPEA, using DMF as a solvent, afforded tetraester $\mathbf{2 0}$ in $26 \%$ yield. Tetraacid $\mathbf{2 1}$ was quantitatively obtained after treatment of 20 with $4 \mathrm{M} \mathrm{HCl}$ in dioxane.

Otherwise, cyclic or open-chain ligands bearing picolinate moieties have shown enhanced ability to coordinate metal cations making them useful for manifold applications including highly stable and 
kinetically inert contrast agents [33,34]. For this reason, we decided to synthesize ligand 25 according to Scheme 4 .
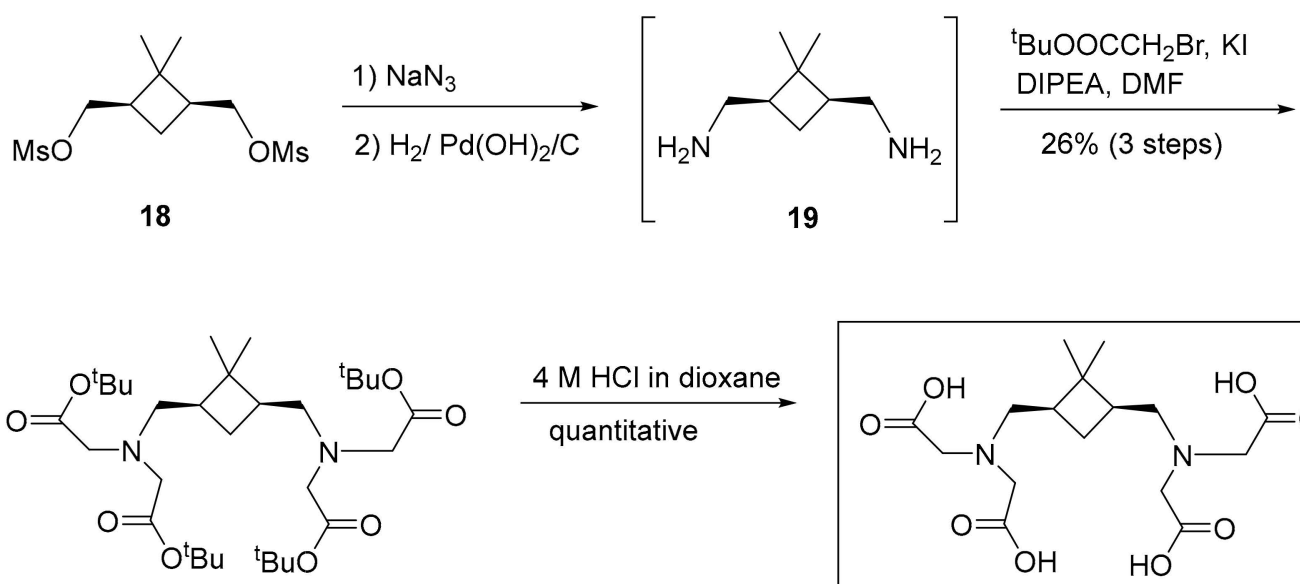

20
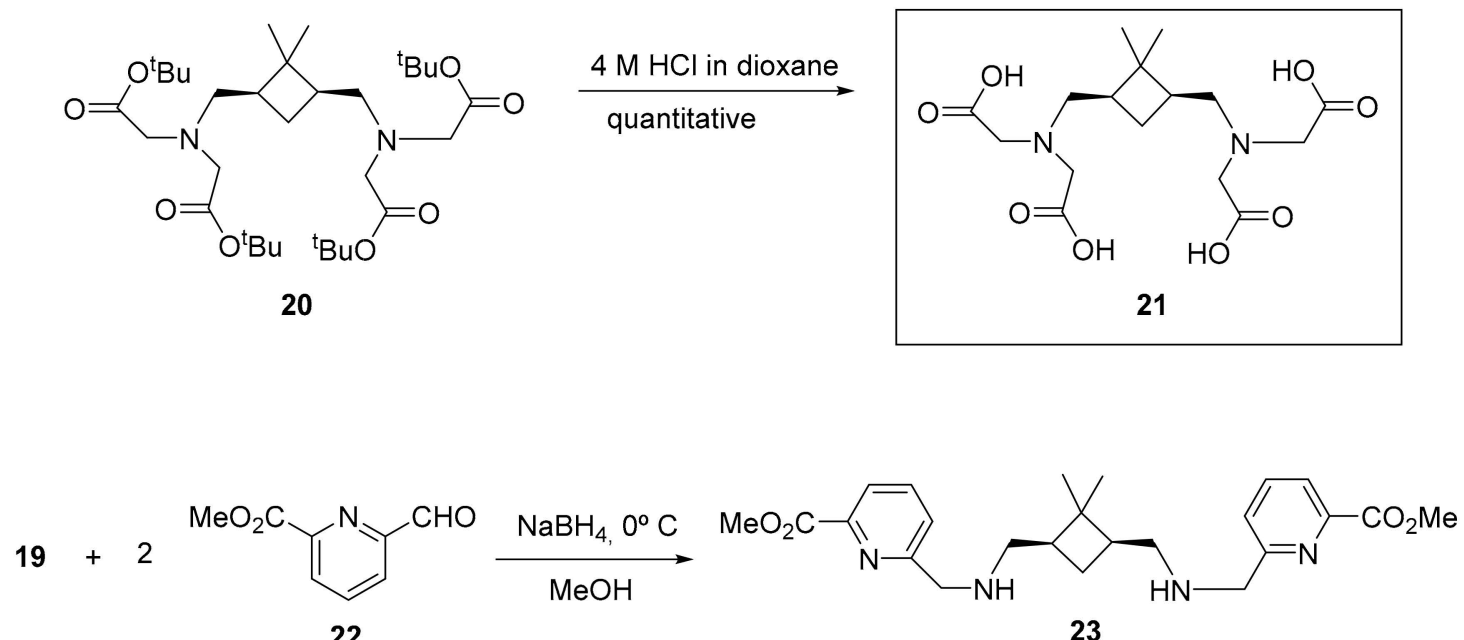

22

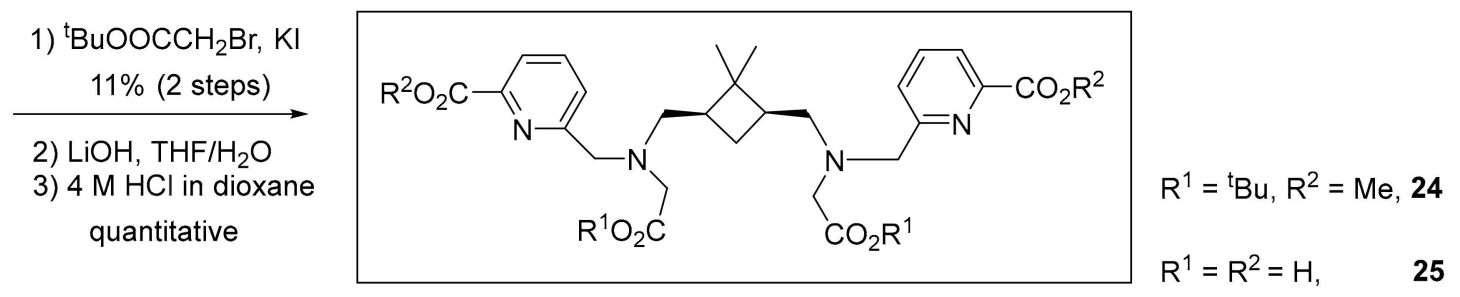

Scheme 4. Synthesis of ligands from 1,3-disubstituted cyclobutane scaffolds.

Methyl 6-formylpicolinate, 22 [37], was submitted to reductive amination by treatment with 0.5 equivalents of diamine 19 and subsequent in situ reduction of the resultant double Schiff-base with sodium borohydride in methanol at $0{ }^{\circ} \mathrm{C}$. Subsequent dialkylation was carried out as described before for the preparation of $\mathbf{2 0}$, giving the analogue $\mathbf{2 4}$ in $11 \%$ yield for the two steps. Finally, saponification of the two methyl esters with 1.2 equivalents of $\mathrm{LiOH}$ in a 1:1 THF/water mixture at room temperature followed by treatment with $4 \mathrm{M} \mathrm{HCl}$ in dioxane, to remove the Boc protecting groups, led to ligand 25 in quantitative yield.

\section{Materials and Methods}

\subsection{General Procedures}

Melting points were determined using a Kofler apparatus model Reicher Austria ${ }^{\mathrm{TM}}$ and are uncorrected. Specific rotations were recorded on a JASCO-715 optical polarimeter. Infrared spectra were recorded on a Bruker Tensor 27 spectrophotometer with sapphire-ATR (Golden Gate). Nuclear magnetic resonance spectra were recorded on an AC 250, AVANCE 360, or ARX Bruker apparatus. Mass spectra (MS) were obtained on a Bruker Squire 3000 micrOTOF spectrometer using ESI-MS (QTOF). Thin-layer chromatography was performed using ALUGRAM ${ }^{\circledR}$ SIL G/UV ${ }_{254}$ aluminium 
sheets pre-coated with silica gel $60(0.20 \mathrm{~mm}$ thickness) containing a fluorescent indicator at $254 \mathrm{~nm}$ (Macherey-Nagel, Düren, Germany). The TLC spots were visualized with a UV lamp or by staining with vanillin in $96 \% \mathrm{EtOH}$ or with an acid $\mathrm{KMnO}_{4}$ solution. Column chromatography was carried out using PanReac ${ }^{\circledR}$ silica gel (230-400 mesh) (Castellar del Vallès, Spain). Nitrogen was used as driving gas.

\subsection{Experimental Section}

\subsubsection{Synthesis of tert-Butyl (1S,2R)-2-Hydroxymethylcyclobutane-1-Carboxylate (2)}

$1 \mathrm{M} \mathrm{B}_{2} \mathrm{H}_{6}$ in THF (10.5 mL, $\left.10.5 \mathrm{mmol}\right)$ was added to a solution of half-ester 1 [14] (1.4 g, $\left.6.9 \mathrm{mmoL}\right)$ in anhydrous THF $(70 \mathrm{~mL})$ and the resultant mixture was stirred at $0{ }^{\circ} \mathrm{C}$ for $2 \mathrm{~h}$ under nitrogen atmosphere and $2 \mathrm{~h}$ at room temperature. The reaction was quenched with aqueous saturated $\mathrm{NH}_{4} \mathrm{Cl}$ $(50 \mathrm{~mL})$, then water $(50 \mathrm{~mL})$ was added and the mixture was extracted with EtOAc $(4 \times 80 \mathrm{~mL})$. The combined organic layers were dried over $\mathrm{MgSO}_{4}$ and solvent was removed at reduced pressure. The residue was purified by flash chromatography (2:1 hexane/EtOAc) to afford alcohol $2(0.85 \mathrm{~g}$, $63 \%$ yield $)$ as an oil. $\mathrm{Rf}=0.27\left(2: 1\right.$ hexane/ethyl acetate); $[\alpha]_{\mathrm{D}}-21.7\left(\mathrm{c}=1.01\right.$ in $\left.\mathrm{CH}_{2} \mathrm{Cl}_{2}\right) ;{ }^{1} \mathrm{H}$ NMR $\left(250 \mathrm{MHz}, \mathrm{CDCl}_{3}\right) \delta=1.42\left(\mathrm{~s}, 9 \mathrm{H}, \mathrm{O}^{\mathrm{t}} \mathrm{Bu}\right) ; 1.68(\mathrm{~m}, 1 \mathrm{H}, \mathrm{H} 3) ; 1.99(\mathrm{~m}, 2 \mathrm{H}, \mathrm{H} 3, \mathrm{H} 4) ; 2.23(\mathrm{~m}, 1 \mathrm{H}, \mathrm{H} 3) ; 2.73$ $(\mathrm{m}, 1 \mathrm{H}, \mathrm{H} 2) ; 3.02(\mathrm{~m}, 1 \mathrm{H}, \mathrm{OH}) ; 3.16(\mathrm{~m}, 1 \mathrm{H}, \mathrm{H} 1) ; 3.58(\mathrm{~m}, 1 \mathrm{H}, \mathrm{H} 5) ; 3.71(\mathrm{~m}, 1 \mathrm{H}, \mathrm{H} 5) \mathrm{ppm} ;{ }^{13} \mathrm{C}$ NMR (62.5 MHz, $\mathrm{CDCl}_{3}$ ) $\delta$ = 21.3 (C3); 22.1 (C4); 28.4 (CBoc); 40.1 (C2); 41.2 (C1); 64.1 (C5); 81.3 (C7); 174.6 (C6) ppm; IR v=3411, 1720, $1152 \mathrm{~cm}^{-1}$; HRMS $\left(\mathrm{ESI}^{+}\right)$calcd $\mathrm{m} / \mathrm{z}$ for $\mathrm{C}_{10} \mathrm{H}_{18} \mathrm{O}_{3}[\mathrm{M}+\mathrm{Na}]^{+}: 209.1148$, found: 209.1152 (Supplementary Materials).

\subsubsection{Synthesis of tert-Butyl (1R,2S)-2-Tosyloxymethylcyclobutane-1-Carboxylate (3)}

A solution containing alcohol 2 ( $0.62 \mathrm{~g}, 3.3 \mathrm{mmol})$, p-toluenesulfonyl chloride $(0.85 \mathrm{~g}, 4.3 \mathrm{mmol})$, 4-DMAP $(0.1 \mathrm{~g}, 0.8 \mathrm{mmoL})$ and TEA $(0.7 \mathrm{~mL}, 5.0 \mathrm{mmoL})$ in dry $\mathrm{CH}_{2} \mathrm{Cl}_{2}(30 \mathrm{~mL})$ was stirred at room temperature overnight under nitrogen atmosphere. Then water $(100 \mathrm{~mL})$ was added and the mixture was extracted with $\mathrm{CH}_{2} \mathrm{Cl}_{2}(3 \times 60 \mathrm{~mL})$. Phases were separated and the organic layer was dried over $\mathrm{MgSO}_{4}$. Solvent was removed and the residue was purified by flash column chromatography (3:1 hexane/EtOAc) to afford tosylate $3(0.87 \mathrm{~g}, 77 \%$ yield $)$ as an oil. $\mathrm{Rf}=0.30(3: 1$ hexane/EtOAc $) ;[\alpha]_{\mathrm{D}}$ $+223.6\left(\mathrm{c}=1.03\right.$ in $\left.\mathrm{CH}_{2} \mathrm{Cl}_{2}\right) ;{ }^{1} \mathrm{H} \mathrm{NMR}\left(360 \mathrm{MHz}, \mathrm{CDCl}_{3}\right) \delta=1.41\left(\mathrm{~s}, 9 \mathrm{H},{ }^{\mathrm{t}} \mathrm{Bu}\right) ; 1.72(\mathrm{~m}, 1 \mathrm{H}, \mathrm{H} 3) ; 2.02$ (m, 2H, H4, H3); 2.23 (m, 1H, H3); 2.44 (s, 3H, H7'); 2.88 (m, 1H, H1); 3.16 (m, 1H, H2); 4.13 (dd, 1H, $\left.\mathrm{H} 5, J=10.5 \mathrm{~Hz}, J^{\prime}=7.2 \mathrm{~Hz}\right) ; 4.21\left(\mathrm{dd}, 1 \mathrm{H}, \mathrm{H} 5, J=10.5 \mathrm{~Hz}, J^{\prime}=7.2 \mathrm{~Hz}\right) ; 7.34\left(\mathrm{~d}, J=11.7 \mathrm{~Hz}, 2 \mathrm{H}, \mathrm{H} 3^{\prime}\right.$, $\left.\mathrm{H} 5^{\prime}\right) ; 7.75\left(\mathrm{~d}, J=11.7 \mathrm{~Hz}, 2 \mathrm{H}, \mathrm{H}^{\prime}{ }^{\prime}, \mathrm{H}^{\prime}\right) ;{ }^{13} \mathrm{C} \mathrm{NMR}\left(90 \mathrm{MHz}, \mathrm{CDCl}_{3}\right) \delta=21.0,21.3,21.4\left(\mathrm{C} 4, \mathrm{C}^{\prime}, \mathrm{C} 3\right)$; 27.7 (CBoc); 35.6 (C1); 39.8 (C2); 70.5 (C5); 80.7 (C7); 127.6 (C2', C6'); 129.5 (C3', C5'); 132.8 (C4'); 144.4 $\left(\mathrm{C}^{\prime}\right) ; 172.1$ (C6); IR $v=2979.3,1721.17,1600.6 \mathrm{~cm}^{-1} ; \mathrm{HRMS}\left(\mathrm{ESI}^{+}\right)$calcd $m / z$ for $\mathrm{C}_{17} \mathrm{H}_{25} \mathrm{NO}_{5} \mathrm{~S}[\mathrm{M}+$ $\mathrm{Na}]^{+}:$363.1237, found: 363.1239 .

3.2.3. Synthesis of tert-Butyl (1S,2R)-2-Dodecanamidomethylcyclobutane-1-Carboxylate (5) through amine 4

A mixture of tosylate $3(0.84 \mathrm{~g}, 2.4 \mathrm{mmol})$ and sodium azide $(0.48 \mathrm{~g}, 7.4 \mathrm{mmol})$ in anhydrous DMF $(30 \mathrm{~mL})$ was stirred and heated at $75^{\circ} \mathrm{C}$ for $3 \mathrm{~h}$ under nitrogen atmosphere. Then EtOAc $(60 \mathrm{~mL})$ was added and the resultant solution was washed with water $(5 \times 50 \mathrm{~mL})$. The combined aqueous layers were extracted with EtOAc $(100 \mathrm{~mL})$ and extract was washed with water $(5 \times 50 \mathrm{~mL})$. The combined organic layers were dried over $\mathrm{MgSO}_{4}$ and concentrated (caution: azides can be explosive, so their solutions must be never evaporated to dryness). The residue was purified by column chromatography (6:1 hexane/ethyl acetate) to afford yellowish oil ( $\mathrm{Rf}=0.44,6: 1$ hexane/ethyl acetate). The obtained oil in THF $(20 \mathrm{~mL})$ was hydrogenated under 1.9 atmospheres pressure in the presence of $20 \% \mathrm{Pd}(\mathrm{OH})_{2} / \mathrm{C}$ as a catalyst $(0.10 \mathrm{~g})$. After $5 \mathrm{~h}$ the mixture was filtered over celite ${ }^{\circledR}$ (Sigma-Aldrich, Darmstadt, Germany), which was washed with methanol $(30 \mathrm{~mL})$. Solvents were removed to afford a solid corresponding 
to partially protected diamine 4 that was used in the next step without further purification due to its instability.

A light protected solution of crude diamine $4(0.10 \mathrm{~g})$, dodecanoyl chloride $(0.12 \mathrm{~mL}, 1.5 \mathrm{mmoL})$ and TEA $(0.16 \mathrm{~mL}, 1.9 \mathrm{mmoL})$ in dry $\mathrm{CH}_{2} \mathrm{Cl}_{2}(10 \mathrm{~mL})$ was stirred overnight under nitrogen atmosphere. The reaction mixture was successively washed with aqueous saturated $\mathrm{NaHCO}_{3}$ and brine. The organic layer was dried over $\mathrm{MgSO}_{4}$ and solvent was evaporated to dryness. The residue was washed with pentane affording compound $5\left(0.1 \mathrm{~g}, 51 \%\right.$ yield for the 3 steps) as a solid. m.p. $55-57^{\circ} \mathrm{C}$ (pentane); $[\alpha]_{\mathrm{D}}+10.7\left(c=1.06\right.$ in $\left.\mathrm{CH}_{2} \mathrm{Cl}_{2}\right) ;{ }^{1} \mathrm{H}$ NMR $\left(250 \mathrm{MHz}, \mathrm{CDCl}_{3}\right) \delta=0.90\left(\mathrm{~m}, 3 \mathrm{H}, \mathrm{H} 12^{\prime}\right) ; 1.28\left(\mathrm{~m}, 16 \mathrm{H}, \mathrm{H} 4^{\prime}\right.$, $\mathrm{H} 5^{\prime}, \mathrm{H}^{\prime}, \mathrm{H}^{\prime}$, $\mathrm{H}^{\prime}$, $\left.\mathrm{H}^{\prime}{ }^{\prime}, \mathrm{H} 10^{\prime}, \mathrm{H}^{\prime} 1^{\prime}\right) ; 1.51$ (s, 9H, $\left.{ }^{\mathrm{t}} \mathrm{Bu}\right) ; 1.64$ (m, 2H, H3'); 1.75 (m, 1H, H3); 2.07 (m, 2H, H3, H4); 2.18 (m, 3H, H2' , H4); 2.80 (m, 1H, H2); 3.19 (m, 2H, H1, H5); 3.56 (m, 1H, H5); 6.10 (broad s, $1 \mathrm{H}, \mathrm{NH}-\mathrm{C}=\mathrm{O}) \mathrm{ppm} ;{ }^{13} \mathrm{C} \mathrm{NMR}\left(62.5 \mathrm{MHz}, \mathrm{CDCl}_{3}\right) \delta=14.6\left(\mathrm{C} 12^{\prime}\right) ; 21.7-32.9\left(\mathrm{C} 3, \mathrm{C} 4, \mathrm{C}^{\prime}, \mathrm{C}^{\prime}, \mathrm{C}^{\prime}{ }^{\prime}, \mathrm{C}^{\prime}\right.$, $\mathrm{C}^{\prime}, \mathrm{C}^{\prime}, \mathrm{C} 8^{\prime}, \mathrm{C}^{\prime}$, $\left.\mathrm{C} 11^{\prime}\right)$ ); 28.7 (CBoc); 37.4, 38.2 (C2' , C2); 41.3, 41.8 (C1, C5); 81.3 (C7); 173.7 (C1'); 174.3 (C6) ppm; IR $v=3328,2916,1715,1644,1153 \mathrm{~cm}^{-1}$; HRMS $\left(\mathrm{ESI}^{+}\right.$) calcd $m / z$ for $\mathrm{C}_{22} \mathrm{H}_{41} \mathrm{NO}_{3}\left[\mathrm{M}+\mathrm{Na}^{+}\right.$: 390.2979, found: 390.2981 .

\subsubsection{Synthesis of (1S,2R)-2-Dodecanamidomethylcyclobutane-1-Carboxylic Acid (6)}

A solution containing ester 5 (90 mg, $0.2 \mathrm{mmol})$, TFA $(0.25 \mathrm{~mL}, 3.8 \mathrm{mmoL})$, and triethylsilane $(0.10 \mathrm{~mL}, 0.6 \mathrm{mmol})$ in dry $\mathrm{CH}_{2} \mathrm{Cl}_{2}(5 \mathrm{~mL})$ was stirred at room temperature overnight under nitrogen atmosphere. Most of solvent was removed and the residue was lyophilized to afford acid 6 (74 mg, quantitative yield) as a solid. m.p. $48-50{ }^{\circ} \mathrm{C} ;[\alpha]_{\mathrm{D}}+19.0\left(\mathrm{c}=1.10\right.$ in $\left.\mathrm{CH}_{2} \mathrm{Cl}_{2}\right) ;{ }^{1} \mathrm{H} \mathrm{NMR}(250 \mathrm{MHz}$, $\left.\mathrm{CDCl}_{3}\right) \delta=0.90\left(\mathrm{t}, 3 \mathrm{H}, \mathrm{H12}{ }^{\prime}, J=6.8 \mathrm{~Hz}\right) ; 1.27\left(\mathrm{~m}, 16 \mathrm{H}, \mathrm{H}^{\prime}{ }^{\prime}, \mathrm{H}^{\prime}{ }^{\prime}, \mathrm{H}^{\prime}, \mathrm{H}^{\prime}, \mathrm{H}^{\prime}, \mathrm{H}^{\prime}, \mathrm{H}^{\prime} 0^{\prime}, \mathrm{H}^{\prime}\right) ; 1.61$ $\left(\mathrm{m}, 2 \mathrm{H}, \mathrm{H} 3^{\prime}\right) ; 1.81(\mathrm{~m}, 1 \mathrm{H}, \mathrm{H} 3) ; 2.12$ (m, 2H, H3, H4); 2.21 (t., $\left.2 \mathrm{H}, \mathrm{H2}{ }^{\prime}, J=6.7 \mathrm{~Hz}\right) ; 2.35$ (m, 1H, H4); $2.90(\mathrm{~m}, 1 \mathrm{H}, \mathrm{H} 2) ; 3.29$ (m, 1H, H5); 3.46 (m, 2H, H5, H1); 6.36 (broad s, 1H, NH-C=O); 7.61 (broad s, $1 \mathrm{H}, \mathrm{HO}-\mathrm{C}=\mathrm{O}) \mathrm{ppm} ;{ }^{13} \mathrm{C} \mathrm{NMR}\left(62.5 \mathrm{MHz}, \mathrm{CDCl}_{3}\right) \delta=14.5\left(\mathrm{C} 12^{\prime}\right) ; 21.2$ (C3); 23.1 (C4); 26.2-32.3 (C3', $\left.\mathrm{C}^{\prime}, \mathrm{C}^{\prime}, \mathrm{C}^{\prime}, \mathrm{C}^{\prime}, \mathrm{C} 8^{\prime}, \mathrm{C} 9^{\prime}, \mathrm{C} 10^{\prime}, \mathrm{C} 11^{\prime}\right) ; 37.0\left(\mathrm{C} 2^{\prime}\right) ; 38.0$ (C2); 40.7 (C5); 41.7 (C1); 175.1 (C1'); 178.6 (C6) ppm; IR $v=3309,1740,1702,1174 \mathrm{~cm}^{-1}$.

\subsubsection{Synthesis of tert-Butyl (1S,2R)-2-Hydroxymethylcyclobutane-1-Carbamate (8)}

A solution of $2 \mathrm{M} \mathrm{LiBH}_{4}$ in THF $(2.6 \mathrm{~mL}, 5.2 \mathrm{mmol})$ was added to a solution of amino ester 7 $\left(1 \mathrm{~g}\right.$, 4.4. mmol) in $\mathrm{Et}_{2} \mathrm{O}(100 \mathrm{~mL})$. The mixture was stirred at $0{ }^{\circ} \mathrm{C}$ for $3 \mathrm{~h}$ under nitrogen atmosphere. Aqueous saturated $\mathrm{NH}_{4} \mathrm{Cl}$ was added $(60 \mathrm{~mL})$ and the resultant mixture was stirred for $30 \mathrm{~min}$, then water $(50 \mathrm{~mL})$ was added and the mixture was consecutively extracted with EtOAc $(3 \times 80 \mathrm{~mL})$; the organic layer was dried over $\mathrm{MgSO}_{4}$ and evaporated to dryness. The residue was chromatographed on silica gel (3:1 hexane/EtOAc) to afford alcohol $8(0.67 \mathrm{~g}, 75 \%)$ as a solid $(\mathrm{Rf}=0.24,3: 1$ hexane/EtOAc); crystals, m.p. $79-81^{\circ} \mathrm{C}$ (hexane/EtOAc); $[\alpha]_{\mathrm{D}}-77.8\left(\mathrm{c}=1.02\right.$ in $\left.\mathrm{CH}_{2} \mathrm{Cl}_{2}\right) ;{ }^{1} \mathrm{H}$ NMR $\left(250 \mathrm{MHz}, \mathrm{CDCl}_{3}\right)$ $\delta=1.46\left(\mathrm{~s}, 9 \mathrm{H},{ }^{\mathrm{t}} \mathrm{Bu}\right) ; 1.63(\mathrm{~m}, 1 \mathrm{H}, \mathrm{H} 3) ; 1.88(\mathrm{~m}, 2 \mathrm{H}, \mathrm{H} 4, \mathrm{H} 3) ; 2.36(\mathrm{~m}, 1 \mathrm{H}, \mathrm{H} 4) ; 2.71(\mathrm{~m}, 1 \mathrm{H}, \mathrm{H} 2) ; 3.63$ $\left(\mathrm{dd}, 1 \mathrm{H}, \mathrm{H} 5, J=11.3 \mathrm{~Hz}, J^{\prime}=4.3 \mathrm{~Hz}\right) ; 3.78\left(\mathrm{dd}, 1 \mathrm{H}, \mathrm{H} 5, J=11.3 \mathrm{~Hz}, J^{\prime}=4.3 \mathrm{~Hz}\right) ; 3.75(\mathrm{~m}, 1 \mathrm{H}) ; 4.21(\mathrm{~m}$, 1H, H1); 5.12 (broad s, 1H, NHBoc) ppm; ${ }^{13} \mathrm{C}$ NMR (62.5 MHz, $\mathrm{CDCl}_{3}$ ) $\delta=19.2$ (C3); 28.8 (C4); 28.7 (CBoc); 41.9 (C2); 48.1 (C1); 62.8 (C5); 80.1 (C7); 156.9 (C6) ppm; IR v = 3311, 2976, 1691, $1174 \mathrm{~cm}^{-1}$; HRMS $\left(\mathrm{ESI}^{+}\right.$) calcd $\mathrm{m} / \mathrm{z}$ for $\mathrm{C}_{10} \mathrm{H}_{19} \mathrm{NO}_{3}[\mathrm{M}+\mathrm{Na}]^{+}:$224.1257, found: 224.1260 .

\subsubsection{Synthesis of (1S,2R)-1-tert-Butyloxycarbonylaminocyclobutane-1-Methyl Dodecanoate (11)}

A solution of alcohol $8(0.3 \mathrm{~g}, 1.5 \mathrm{mmoL})$, lauryl chloride $(0.36 \mathrm{~mL}, 1.5 \mathrm{mmoL})$, and TEA $(0.27 \mathrm{~mL}$, $1.9 \mathrm{mmoL})$ in dry $\mathrm{CH}_{2} \mathrm{Cl}_{2}(15 \mathrm{~mL})$ was heated to reflux overnight under nitrogen atmosphere. The reaction mixture was cooled to room temperature, washed once with aqueous saturated $\mathrm{NaHCO}_{3}$ $(10 \mathrm{~mL})$ and twice with brine, and dried over $\mathrm{MgSO}_{4}$. Solvent was removed and the residue was purified by flash chromatography $\left(\mathrm{CH}_{2} \mathrm{Cl}_{2}\right)$ to give pure $11(0.4 \mathrm{~g}, 68 \%)$ as a solid. m.p. $44-46{ }^{\circ} \mathrm{C}$ $\left(\mathrm{CH}_{2} \mathrm{Cl}_{2}\right) ;[\alpha]_{\mathrm{D}}-63.9\left(\mathrm{c}=0.97\right.$ in $\left.\mathrm{CH}_{2} \mathrm{Cl}_{2}\right) ;{ }^{1} \mathrm{H} \mathrm{NMR}\left(360 \mathrm{MHz}, \mathrm{CDCl}_{3}\right) \delta=0.87\left(\mathrm{t}, 3 \mathrm{H}, \mathrm{H} 12^{\prime}, J=8.4 \mathrm{~Hz}\right)$;

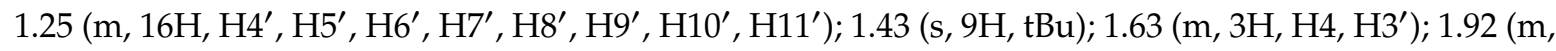
2H, H3, H4); 2.33 (m; 3H, H2' , H3); 2.81 (m, 1H, H1); 4.16 (dd, 1H, H5, J = 13.8 Hz, J' = 7.2 Hz); 4.31 (dd, 
$\left.1 \mathrm{H}, \mathrm{H} 5, J=13.8 \mathrm{~Hz}, J^{\prime}=7.2 \mathrm{~Hz}\right) ; 4.30$ (m, 1H, H2); 4.79 (broad s, $\left.1 \mathrm{H}, \mathrm{NHBoc}\right) \mathrm{ppm} ;{ }^{13} \mathrm{C} \mathrm{NMR}(90 \mathrm{MHz}$, $\left.\mathrm{CDCl}_{3}\right) \delta=13.8\left(\mathrm{C} 12^{\prime}\right) ; 17.7(\mathrm{C} 4) ; 22.2-31.6\left(\mathrm{C}^{\prime}, \mathrm{C}^{\prime}, \mathrm{C}^{\prime}, \mathrm{C}^{\prime}, \mathrm{C}^{\prime}, \mathrm{C} 8^{\prime}, \mathrm{C} 9^{\prime}, \mathrm{C} 10^{\prime}, \mathrm{C} 11^{\prime}\right) ; 26.6(\mathrm{C} 4) ; 28.1$ (CBoc); 34.1 (C2'); 38,6 (C1); 45.8 (C2); 63.4 (C5); 79.0 (C7); 154.7 (C6); 173.7 (C1') ppm; IR v = 3342, 2919, 1679, $1166 \mathrm{~cm}^{-1}$; HRMS (ESI ${ }^{+}$) calcd m/z for $\mathrm{C}_{22} \mathrm{H}_{41} \mathrm{NO}_{4}[\mathrm{M}+\mathrm{Na}]^{+}: 406.2928$, found: 406.2930 .

\subsubsection{Synthesis of $(1 S, 2 R)-2$-Dodecanoyloxymethylcyclobutane-1-Ammonium Chloride (12)}

A solution of compound $11(0.2 \mathrm{~g}, 0.5 \mathrm{mmol})$ and $2 \mathrm{~N} \mathrm{HCl}$ in $\mathrm{Et}_{2} \mathrm{O}(1.56 \mathrm{~mL}, 3.1 \mathrm{mmol})$ in dry $\mathrm{CH}_{2} \mathrm{Cl}_{2}(15 \mathrm{~mL})$ was stirred at room temperature for $24 \mathrm{~h}$ under nitrogen atmosphere. Most solvent was removed, and the residue was lyophilized to afford quantitatively a solid that was crystallized in pentane to provide surfactant $12\left(0.14 \mathrm{~g}, 84 \%\right.$ yield) as crystals. m.p. $86-88^{\circ} \mathrm{C}$ (pentane); $[\alpha]_{\mathrm{D}}+9.6$ (c = 1.01 in $\mathrm{MeOH}) ;{ }^{1} \mathrm{H}$ NMR $\left(360 \mathrm{MHz}, \mathrm{CD}_{3} \mathrm{OD}\right) \delta=0.91\left(\mathrm{~m}, 3 \mathrm{H}, \mathrm{H} 12^{\prime}\right) ; 1.30\left(\mathrm{~m}, 16 \mathrm{H}, \mathrm{H}^{\prime}, \mathrm{H}^{\prime}, \mathrm{H}^{\prime}\right.$, $\mathrm{H}^{\prime}, \mathrm{H}^{\prime}, \mathrm{H}^{\prime}$, H10', H11'); 1.63 (m, 2H, H3'); 1.81 (m, 1H, H3); 2.17 (m, 2H, H4, H3); 2.39 (m, 3H, $\left.\mathrm{H} 2^{\prime}, \mathrm{H} 4\right) ; 2.95$ (m, 1H, H2); 3.95 (m, 1H, H1); 4.32 (m, 2H, H5); $\left.{ }^{13} \mathrm{C} \mathrm{NMR} \mathrm{(90} \mathrm{MHz,} \mathrm{CD}{ }_{3} \mathrm{OD}\right) \delta=13.1$

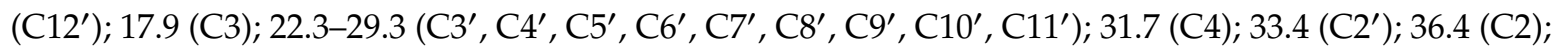
46.6 (C5); 62.3 (C1); 173.8 (C1') ppm; IR $v=3370,2919,1740,1112 \mathrm{~cm}^{-1}$; HRMS (ESI ${ }^{+}$) calcd $\mathrm{m} / z$ for $\mathrm{C}_{17} \mathrm{H}_{34} \mathrm{ClNO}_{2}[\mathrm{M}]^{+}:$319.2348, found: 319.2346 .

\subsubsection{Synthesis of tert-Butyl (1S,2R)-2-Tosyloxymethylcyclobutane-1-Carboxylate (9)}

Following a procedure similar to that described above for the preparation of tosylate 3 , compound $9(0.57 \mathrm{~g}, 62 \%$ yield $)$ was obtained as a solid. $\mathrm{Rf}=0.28$ (6:1 hexane/EtOAc); crystals, m.p. $87-88^{\circ} \mathrm{C}$ (ethyl acetate/hexane); $[\alpha]_{\mathrm{D}}-102.9\left(\mathrm{c}=1.05\right.$ in $\left.\mathrm{CH}_{2} \mathrm{Cl}_{2}\right) ;{ }^{1} \mathrm{H}$ NMR $\left(360 \mathrm{MHz}, \mathrm{CDCl}_{3}\right) \delta=1.41(\mathrm{~s}, 9 \mathrm{H}$, $\left.{ }^{\mathrm{t}} \mathrm{Bu}\right) ; 1.58(\mathrm{~m}, 1 \mathrm{H}, \mathrm{H} 3) ; 1.88$ (m, 2H, H4, H3); 2.27 (m, 1H, H3); 2.45 (s, 3H, H7'); 2.75 (m, 1H, H1); 4.17 (m, 2H, H5); 4.33 (m, 1H, H2); 4.77 (broad s, 1H, NHBoc); 7.34 (d, J = 11.7 Hz, 2H, H3' , H5'); 7.75 (d, $\left.J=11.7 \mathrm{~Hz}, 2 \mathrm{H}, \mathrm{H2}{ }^{\prime}, \mathrm{H6}^{\prime}\right) \mathrm{ppm} ;{ }^{13} \mathrm{C}$ NMR $\left(90 \mathrm{MHz}, \mathrm{CDCl}_{3}\right) \delta=17.6(\mathrm{C} 4) ; 21.4\left(\mathrm{C}^{\prime}\right) ; 28.0$ (CBoc); 28.1 (C3); 38.7 (C1); 45.8 (C2); 69.8 (C5); 79.1 (C7); $127.6\left(\mathrm{C} 2^{\prime}, \mathrm{C}^{\prime}\right)$; $129.6\left(\mathrm{C}^{\prime}, \mathrm{C}^{\prime}\right) ; 132.6\left(\mathrm{C} 4^{\prime}\right) ; 144.6\left(\mathrm{C} 1^{\prime}\right)$; 154.8 (C6) ppm; IR: $v=3311,2976,1691,1174 \mathrm{~cm}^{-1}$; HRMS $\left(\mathrm{ESI}^{+}\right)$calcd m/z for $\mathrm{C}_{17} \mathrm{H}_{25} \mathrm{NO}_{5} \mathrm{~S}[\mathrm{M}+$ $\mathrm{Na}^{+}:$378.1346, found: 378.1344 .

3.2.9. Synthesis of tert-Butyl (1S,2S)-2-Dodecanamidomethylcyclobutane-1-Carbamate (13) through diamine 10

Following a similar procedure than that described above for the preparation of 5, compound 13 (66\% overall yield for the three steps) was obtained as crystals. m.p. $102-104{ }^{\circ} \mathrm{C}$ (pentane); $[\alpha]_{\mathrm{D}}-40.2$ $\left(\mathrm{c}=1.01\right.$ in $\left.\mathrm{CH}_{2} \mathrm{Cl}_{2}\right) ;{ }^{1} \mathrm{H}$ NMR $\left(360 \mathrm{MHz}, \mathrm{CDCl}_{3}\right) \delta=0.89\left(\mathrm{t}, 3 \mathrm{H}, \mathrm{H} 12^{\prime}, \mathrm{J}=5.7 \mathrm{~Hz}\right) ; 1.25\left(\mathrm{~m}, 16 \mathrm{H}, \mathrm{H} 4^{\prime}\right.$, $\mathrm{H}^{\prime}, \mathrm{H6}^{\prime}, \mathrm{H7} 7^{\prime}, \mathrm{H}^{\prime}$, H9' , H10', H11'); 1.44 (s, 9H, tBu); 1.61 (m, 3H, H3, H3'); 1.80 (m, 1H, H4); 1.92 (m, 1H, H3); 2.13 (t., 2H, H2' , J = 6.3 Hz); 2.36 (m, 1H, H4); 2.59 (m, 1H, H2); 3.15 (m, 1H, H5); 3.52 (m, 1H, $\mathrm{H} 5) ; 4.17$ (m, 1H, H1); 4.99 (broad s, 1H, NHBoc); 6.12 (broad s, 1H, NH-C=O) ppm; ${ }^{13} \mathrm{C}$ NMR $(90 \mathrm{MHz}$, $\left.\mathrm{CDCl}_{3}\right) \delta=14.1\left(\mathrm{C} 12^{\prime}\right) ; 20.1(\mathrm{C} 3) ; 26.6(\mathrm{C} 4) ; 22.7-29.6\left(\mathrm{C2}^{\prime}, \mathrm{C}^{\prime}, \mathrm{C}^{\prime}, \mathrm{C}^{\prime}, \mathrm{C}^{\prime}, \mathrm{C}^{\prime}, \mathrm{C}^{\prime}, \mathrm{C}^{\prime}, \mathrm{C} 11^{\prime}\right) ; 28.4$ (CBoc); 36.9 (C10'); 39.1-40.0 (C2, C5); 47.2 (C1); 79.7 (C7); 156.2 (C6); 173.3 (C1') ppm; IR v = 3342, $2919,1679 \mathrm{~cm}^{-1}$; HRMS $\left(\mathrm{ESI}^{+}\right.$) calcd $m / z$ for $\mathrm{C}_{22} \mathrm{H}_{42} \mathrm{~N}_{2} \mathrm{O}_{3}[\mathrm{M}+\mathrm{Na}]^{+}: 405.3088$, found: 405.3088 .

\subsubsection{Synthesis of (1S,2R)-2-Dodecanamidomethylcyclobutane-1-Ammonium Chloride (14)}

A solution of compound $13(0.2 \mathrm{~g}, 0.5 \mathrm{mmol})$ and $2 \mathrm{~N} \mathrm{HCl}$ in $\mathrm{Et}_{2} \mathrm{O}(1.56 \mathrm{~mL}, 3.1 \mathrm{mmol})$ in dry $\mathrm{CH}_{2} \mathrm{Cl}_{2}(15 \mathrm{~mL})$ was stirred at room temperature for $24 \mathrm{~h}$ under nitrogen atmosphere. Most solvent was removed, and the residue was lyophilized to afford quantitatively a solid that was crystallized in pentane to provide amphiphile $14\left(0.1 \mathrm{~g}, 64 \%\right.$ yield). Crystals, m.p. $98-100{ }^{\circ} \mathrm{C}$ (pentane); $[\alpha]_{\mathrm{D}}+8.7$ $(\mathrm{c}=1.05 \mathrm{in} \mathrm{MeOH}) ;{ }^{1} \mathrm{H}$ NMR $\left(360 \mathrm{MHz}, \mathrm{CD}_{3} \mathrm{OD}\right) \delta=0.91\left(\mathrm{~m}, 3 \mathrm{H}, \mathrm{H} 12^{\prime}\right) ; 1.30\left(\mathrm{~m}, 16 \mathrm{H}, \mathrm{H}^{\prime}, \mathrm{H}^{\prime}, \mathrm{H}^{\prime}\right.$, $\left.\mathrm{H} 7^{\prime}, \mathrm{H}^{\prime}, \mathrm{H}^{\prime}, \mathrm{H} 10^{\prime} \mathrm{H} 11^{\prime}\right) ; 1.61$ (m, 2H, H3'); 1.98 (m, 2H, H3, H4); 2.09 (m, 1H, H3); 2.23 (t, 2H, H2', $\mathrm{J}=6.3 \mathrm{~Hz}) ; 2.39$ (m, 1H, H4); 2.79 (m, 1H, H1); 3.16 (m, 1H, H5); 3.44 (m, 1H, H5); 3.75 (m, 1H, H1) ppm; ${ }^{13} \mathrm{C}$ NMR $\left(90 \mathrm{MHz}, \mathrm{CD}_{3} \mathrm{OD}\right) \delta=13.0\left(\mathrm{C} 12^{\prime}\right) ; 20.2$ (C3); $22.3(\mathrm{C} 4) ; 22.7-29.6\left(\mathrm{C}^{\prime}, \mathrm{C}^{\prime}, \mathrm{C} 5^{\prime}, \mathrm{C}^{\prime}, \mathrm{C}^{\prime}, \mathrm{C} 8^{\prime}\right.$, 
$\left.\mathrm{C}^{\prime}, \mathrm{C} 10^{\prime}, \mathrm{C} 11^{\prime}\right)$; $35.5\left(\mathrm{C} 2^{\prime}\right) ; 37.8-38.6(\mathrm{C} 2, \mathrm{C} 5) ; 47.2(\mathrm{C} 1)$; $176.2\left(\mathrm{C1}^{\prime}\right) \mathrm{ppm}$; IR $v=3342,2918,1679 \mathrm{~cm}^{-1}$; HRMS (ESI ${ }^{+}$) calcd $\mathrm{m} / \mathrm{z}$ for $\mathrm{C}_{17} \mathrm{H}_{35} \mathrm{~N}_{2} \mathrm{O}[\mathrm{M}]^{+}: 283.2744$, found: 283.2745 .

\subsubsection{Synthesis of Diamides 16 and 17. General Procedure}

To a solution of compound 15 [15] $(0.18 \mathrm{~g}, 0.84 \mathrm{mmol})$ in anhydrous $\mathrm{CH}_{2} \mathrm{Cl}_{2}(27.0 \mathrm{~mL})$, DIPEA $(0.44 \mathrm{~mL}, 2.52 \mathrm{mmoL})$ and PyBOP $(0.55 \mathrm{~g}, 1.06 \mathrm{mmoL})$ were added. After $15 \mathrm{~min}$ stirring at room temperature, dodecylamine or hexadecylamine $(0.92 \mathrm{mmoL})$ dissolved in anhydrous $\mathrm{CH}_{2} \mathrm{Cl}_{2}(10.0 \mathrm{~mL})$ was added and the resulting mixture was stirred at room temperature for $18 \mathrm{~h}$. The solution was diluted with $\mathrm{CH}_{2} \mathrm{Cl}_{2}(10.0 \mathrm{~mL})$ and washed with aqueous saturated $\mathrm{NaHCO}_{3}(30.0 \mathrm{~mL})$. The organic layer was dried over $\mathrm{MgSO}_{4}$ and evaporated under vacuum. The residue was purified by column chromatography (1:1 hexane-EtOAc) to afford the corresponding pure amide. A mixture containing this compound $(0.40 \mathrm{mmoL}), 2 \mathrm{~N} \mathrm{HCl}$ in $\mathrm{Et}_{2} \mathrm{O}(20 \mathrm{mmoL})$ and anhydrous $\mathrm{CH}_{2} \mathrm{Cl}_{2}(10.0 \mathrm{~mL})$ was stirred at room temperature for $36 \mathrm{~h}$. The solvent was evaporated at reduced pressure to afford the corresponding amine hydrochloride, which was dissolved in a $1: 1$ mixture of $0.25 \mathrm{M} \mathrm{NaOH}-\mathrm{CH}_{2} \mathrm{Cl}_{2}$ and stirred vigorously for $15 \mathrm{~min}$. Then the reaction mixture was extracted with $\mathrm{CH}_{2} \mathrm{Cl}_{2}(3 \times 10 \mathrm{~mL})$. The combined organic layers were dried over $\mathrm{MgSO}_{4}$ and solvent was removed to afford the respective free amine. This was coupled with lauryl or palmitic acid, respectively, as described above for the first coupling procedure. After purification by column chromatography $\left(5 \% \mathrm{MeOH}-\mathrm{CH}_{2} \mathrm{Cl}_{2}\right)$ the corresponding pure diamides were obtained as solid products.

\section{(1S,2S)-N-Dodecyl-2-Dodecanamidocyclobutane-1-Carboxamide (16)}

$27 \%$ overall yield from 15. Crystals, m.p. $74-79{ }^{\circ} \mathrm{C}\left(\mathrm{CH}_{2} \mathrm{Cl}_{2}\right) ;[\alpha]_{\mathrm{D}}+2.1\left(\mathrm{c}=1.05\right.$ in $\left.\mathrm{CH}_{2} \mathrm{Cl}_{2}\right) ;{ }^{1} \mathrm{H}$ $\operatorname{NMR}\left(360 \mathrm{MHz}, \mathrm{CDCl}_{3}\right) \delta=0.92\left(\mathrm{t}, J=6.4 \mathrm{~Hz}, 6 \mathrm{H}, \mathrm{H} 19, \mathrm{H} 18^{\prime}\right), 1.28\left(\mathrm{~s}, 34 \mathrm{H}, \mathrm{H} 10-18, \mathrm{H} 10^{\prime}-17^{\prime}\right), 1.60(\mathrm{~m}$, 4H, H9, H9'), 1.81-2.00 (m, 2H, H4-3), 2.19 (m, 4H, H3-4, H8'), 2.90 (q, J = 9.1 Hz, 1H, H5), 3.24 (m, 2H, H8), 4.33 (m, 1H, H2), 5.88 (broad s, $1 \mathrm{H}, \mathrm{N} 1 \mathrm{H}), 8.36$ (broad s, $1 \mathrm{H}, \mathrm{N} 7 \mathrm{H}) \mathrm{ppm}$; ${ }^{13} \mathrm{C}$ NMR $(90 \mathrm{MHz}$, $\left.\mathrm{CDCl}_{3}\right) \delta=13.8\left(\mathrm{C} 19, \mathrm{C} 19^{\prime}\right) ; 18.6(\mathrm{C} 4) ; 22.3\left(\mathrm{C} 18, \mathrm{C} 18^{\prime}\right) ; 24.1$ (C9-16,C10'-16'); $25.4\left(\mathrm{C} 9-16, \mathrm{C}^{\prime} 0^{\prime}-16^{\prime}\right)$; 26.8 (C3); 29.0-29.4 (C9-16,C10'-16'); 31.7 (C17,C17'); 36.1 (C17, C17'); 39.1 (C8); 47.7 (C2); 49.7 (C5, C9'); 172.5 (C7); $174.1\left(\mathrm{C}^{\prime}\right)$ ppm; IR $v=2952,1685,1638 \mathrm{~cm}^{-1}$; HRMS $\left(\mathrm{ESI}^{+}\right)$calcd $m / z$ for $\mathrm{C}_{29} \mathrm{H}_{56} \mathrm{~N}_{2} \mathrm{O}_{2}[\mathrm{M}+$ $\mathrm{H}]^{+}:$465.4415, found: 465.4397 .

\section{(1S,2S)-N-Hexadecyl-2-Palmitamidocyclobutane-1-Carboxamide (17)}

$28 \%$ overall yield from 15. Crystals, m.p. $116-118{ }^{\circ} \mathrm{C}\left(\mathrm{CH}_{2} \mathrm{Cl}_{2}\right) ;[\alpha]_{\mathrm{D}}+6.0\left(c=1.04\right.$ in $\left.\mathrm{CH}_{2} \mathrm{Cl}_{2}\right) ;{ }^{1} \mathrm{H}$ NMR $\left(360 \mathrm{MHz}, \mathrm{CDCl}_{3}\right) \delta=0.88\left(\mathrm{t}, J=5.5 \mathrm{~Hz}, 6 \mathrm{H}, \mathrm{H} 23, \mathrm{H} 22^{\prime}\right), 1.26\left(\mathrm{~s}, 50 \mathrm{H}, \mathrm{H} 10-22, \mathrm{H} 10^{\prime}-21^{\prime}\right), 1.61(\mathrm{~m}$, $\left.4 \mathrm{H}, \mathrm{H} 9, \mathrm{H}^{\prime}\right), 1.77-2.00$ (m, 2H, H4-3), 2.18 (m, 4H, H3-4, H8'), 2.89 (q, J = 8.7 Hz, 1H, H5), 3.21 (m, 2H, $\mathrm{H} 8), 4.32(\mathrm{~m}, 1 \mathrm{H}, \mathrm{H} 2), 5.80(\mathrm{~d}, J=6.6 \mathrm{~Hz}, 1 \mathrm{H}, \mathrm{N} 1 \mathrm{H}), 8.34(\mathrm{~s}, 1 \mathrm{H}, \mathrm{N} 7 \mathrm{H}) \mathrm{ppm} ;{ }^{13} \mathrm{C} \mathrm{NMR}\left(90 \mathrm{MHz}, \mathrm{CDCl}_{3}\right)$ $\delta=13.9\left(\mathrm{C} 23, \mathrm{C} 23^{\prime}\right) ; 18.6$ (C4); $22.2\left(\mathrm{C} 22, \mathrm{C} 22^{\prime}\right) ; 24.1\left(\mathrm{C} 9-20, \mathrm{C} 10^{\prime}-20^{\prime}\right) ; 25.4\left(\mathrm{C} 9-20, \mathrm{C} 10^{\prime}-20^{\prime}\right) ; 26.8(\mathrm{C} 3)$; 29.0-29.4 (C9-20, C10'-20'); 31.6 (C21, C21'); 39.3 (C8); 47.4 (C2); 49.7 (C5, C9'); 172.2 (C7); 173.9 (C8') ppm; IR $v=3293,2955,2849,1686,1644 \mathrm{~cm}^{-1}$; HRMS $\left(\mathrm{ESI}^{+}\right)$calcd $m / z$ for $\mathrm{C}_{37} \mathrm{H}_{72} \mathrm{~N}_{2} \mathrm{O}_{2}[\mathrm{M}+\mathrm{Na}]^{+}$: 599.5486, found: 599.5478 .

\subsubsection{Synthesis of Tetra-tert-Butyl $N, N, N^{\prime}, N^{\prime}-[((1 S, 3 R)-2,2-$ Dimethylcyclobutane-1,3-diyl)}

Bis(Methylene)Bis(Azanetriyl)] Tetraacetate (20)

Diamine 19 was prepared from dimesylate 18 [31] as described above for the synthesis of diamine 4 and used in the next step without further purification. tert-Butyl bromoacetate $(3.7 \mathrm{~mL}, 25.1 \mathrm{mmoL})$ was added dropwise to a mixture of crude diamine $19(0.45 \mathrm{~g}, 3.2 \mathrm{mmoL})$, potassium iodide $(2.8 \mathrm{~g}, 17$ $\mathrm{mmoL})$, and DIPEA $(8.7 \mathrm{~mL}, 50.2 \mathrm{mmoL})$ in anhydrous DMF $(15 \mathrm{~mL})$. The resultant solution was stirred at room temperature for $96 \mathrm{~h}$ under nitrogen atmosphere. The mixture was diluted with dichloromethane $(150 \mathrm{~mL})$ and washed with sat. $\mathrm{K}_{2} \mathrm{CO}_{3}(2 \times 30 \mathrm{~mL})$ and brine $(40 \mathrm{~mL})$. The organic phase was dried over $\mathrm{MgSO}_{4}$ and the solvent was evaporated to afford a yellow oil. The crude product was purified by column chromatography (4:1 Hexane/EtOAc) to obtain a colourless oil $(0.48 \mathrm{~g}, 26 \%$ overall yield from dimesylate 
18). ${ }^{1} \mathrm{H}$ NMR (250 MHz, $\left.\mathrm{CDCl}_{3}\right) \delta=0.92$ (s, 3H, H6/5); 1.09 (s, 3H, H5/6); 1.47 (s, 36H, H11); 2.06 (m, 3H, $\mathrm{H} 1, \mathrm{H} 3, \mathrm{H} 4) ; 2.66$ (m, 4H, H7); 3.39 (s, 8H, H8); ppm; ${ }^{13} \mathrm{C} \mathrm{NMR}\left(62.5 \mathrm{MHz}, \mathrm{CDCl}_{3}\right) \delta=16.0$ (C6); 28.0 (C5); 29.6 (C11); 30.0 (C2); 40.0 (C4); 40.9 (C1, C3); 54.5 (C7); 55.9 (C8); 80.8 (C10); 170.7 (C9) ppm; IR v = 1727, $1146 \mathrm{~cm}^{-1}$; HRMS (ESI ${ }^{+}$) calcd $m / z$ for $\mathrm{C}_{32} \mathrm{H}_{58} \mathrm{~N}_{2} \mathrm{O}_{8}[\mathrm{M}+\mathrm{H}]^{+}:$599.4266, found: 599.4252.

3.2.13. Synthesis of $N, N, N^{\prime}, N^{\prime}-[((1 S, 3 R)-2,2$-dimethylcyclobutane-1,3-diyl)

bis(methylene)-bis(azanetriyl)] tetraacetic acid (21)

A solution of tetraester $20(0.20 \mathrm{~g}, 0.3 \mathrm{mmoL})$ in $4 \mathrm{M} \mathrm{HCl}$ in dioxane $(12 \mathrm{~mL}, 48 \mathrm{mmoL})$ was stirred at room temperature for $20 \mathrm{~h}$. Solvent was removed at reduced pressure, then water $(2 \mathrm{~mL})$ was added and the mixture was lyophilized to afford 21 as a solid $\left(0.13 \mathrm{~g}\right.$, quantitative). ${ }^{1} \mathrm{H}$ NMR $(250 \mathrm{MHz}$, $\left.\mathrm{CDCl}_{3}\right) \delta=0.85(\mathrm{~s}, 3 \mathrm{H}, \mathrm{H} 5 / 6) ; 1.03(\mathrm{~s}, 3 \mathrm{H}, \mathrm{H} 5 / 6), 1.14-1.29$ (m, 1H, H4); 1.94-2.11 (m, 3H, H1,3,4); 2.48 (m, 2H, H7); 2.62 (m, 2H, H7); 3.95 (s, 6H, H15); 3.77-4.06 (m, 4H, H8); 7.54 (m, 2H, H9); 7.77 (m, 2H, H10); 7.97 (m, 2H, H11) ppm; ${ }^{13} \mathrm{C}$ NMR (62.5 MHz, $\left.\mathrm{CDCl}_{3}\right) \delta=15.1$ (C6); 27.6 (C5); 29.5 (C4); 37.0 (C2); 41.2 (C3); 55.5 (C7); 56.9 (C8); 168.7 (C9) ppm; IR $v=1730 \mathrm{~cm}^{-1}$; HRMS (ESI ${ }^{+}$) calcd $\mathrm{m} / z$ for $\mathrm{C}_{16} \mathrm{H}_{26} \mathrm{~N}_{2} \mathrm{O}_{8}[\mathrm{M}-\mathrm{H}]^{-}$: 373.1616, found: 373.1626 .

3.2.14. Synthesis of Dimethyl 6,6'-[\{2,2-Dimethylcyclobutane-1,3-diylbis[(2-tert-butoxy-2-oxoethyl)azanemethylenediyl]\}bis(methylene)] dipicolinate (24) through compound (23)

A solution of diamine 19 (0.6 g, $4.3 \mathrm{mmol})$ and methyl 6-formyl picolinate 22 [37] (0.14 g, 8.6 $\mathrm{mmoL})$ in $\mathrm{MeOH}(20 \mathrm{~mL})$ was stirred at room temperature for $5 \mathrm{~h}$. Then, the solution was cooled with ice and $\mathrm{NaBH}_{4}(0.26 \mathrm{~g}, 7 \mathrm{mmoL})$ and $\mathrm{MeOH}(20 \mathrm{~mL})$ were added. The resultant ice-cooled mixture was stirred for $2 \mathrm{~h}$. Aqueous saturated $\mathrm{NaHCO}_{3}(30 \mathrm{~mL})$ was added and most methanol was removed. The solution was extracted with $\mathrm{CH}_{2} \mathrm{Cl}_{2}(4 \times 50 \mathrm{~mL})$, the combined organic layers were dried over $\mathrm{MgSO}_{4}$ and solvent was removed to afford crude compound 23 that was identified by its ${ }^{1} \mathrm{H}$ NMR spectrum and used in the next step without further purification. ${ }^{1} \mathrm{H} \mathrm{NMR}\left(250 \mathrm{MHz}, \mathrm{CDCl}_{3}\right) \delta=0.91$ (s, 3H, H4/5); 1.09 (s, 3H), 1.14-1.29 (m, 1H, H1); 1.94-2.11 (m, 3H, H1,2,3); 2.48 (m, 2H, H6/7); 2.62 (m, 2H, H6/7); 3.95 (s, 6H, H12); 3.77-4.06 (m, 4H, H8); 7.54 (d, J = $7.8 \mathrm{~Hz}, 2 \mathrm{H}, \mathrm{H9} / 11) ; 7.77$ (t, $J=7.7 \mathrm{~Hz}$, 2H, H10); 7.97 (d, $J=7.7 \mathrm{~Hz}, 2 \mathrm{H}, \mathrm{H} 9 / 11)$.

Using the same procedure as that described above to prepare $\mathbf{2 0}$, crude $\mathbf{2 3}(0.5 \mathrm{~g})$ was made to react with tert-butyl bromoacetate affording a reaction crude, which was purified by column chromatography (3:1 to 1:1 hexane/EtOAc) affording tetraester $24(0.35 \mathrm{~g}, 11 \%$ yield for the two steps) as a yellowish oil. ${ }^{1} \mathrm{H}$ NMR $\left(400 \mathrm{MHz}, \mathrm{CDCl}_{3}\right) \delta=0.63(\mathrm{~s}, 3 \mathrm{H}, \mathrm{H} 5 / 6) ; 0.88(\mathrm{~s}, 3 \mathrm{H}, \mathrm{H} 5 / 6) ; 1.13(\mathrm{~m}, 1 \mathrm{H}, \mathrm{H} 4) ; 1.28(\mathrm{~s}, 18 \mathrm{H}$, H19); 1.88 (m, 3H, H1,3,4); 2.44 (m, 4H, H7); 3.07 (m, 4H, H8/16); 3.79 (m, 4H, H8/16); 3.82 (s, 6H, H15); 7.68 (m, 4H, H10,11); 7.84 (m, 2H, H12) ppm; ${ }^{13} \mathrm{C} \mathrm{NMR}\left(62.5 \mathrm{MHz}, \mathrm{CDCl}_{3}\right) \delta=15.8$ (C5,6); 27.9 (C19); 29.3 (C4); 29.9 (C5,6); 39.6 (C3/11); 40.3 (C2); 40.5 (C1,3); 52.5 (C7); 54.8 (C15); 56.1 (C16); 60.2 (C8); 80.6 (C18); 123.2 (C10); 125.7 (C12); 137.0 (C11); 146.8 (C13); 160.9 (C9); 165.6 (C14); 170.2 (C17) ppm; IR $v=2951,2862,2360,1723,1139 \mathrm{~cm}^{-1}$; HRMS $\left(\mathrm{ESI}^{+}\right)$calcd $m / z$ for $\mathrm{C}_{36} \mathrm{H}_{52} \mathrm{~N}_{4} \mathrm{O}_{8}[\mathrm{M}+\mathrm{H}]^{+}: 669.3858$, found: 669.3853 .

3.2.15. Synthesis of 6,6'-[\{2,2-dimethylcyclobutane-1,3-diylbis[(carboxymethyl) azane methylene diyl]\}bis(methylene)] dipicolinic acid (25)

A mixture containing compound $24(200 \mathrm{mg}, 0.30 \mathrm{mmol})$ and $\mathrm{LiOH}(45 \mathrm{mg}, 1.19 \mathrm{mmoL})$ in $1: 1$ $\mathrm{THF} / \mathrm{H}_{2} \mathrm{O}(5 \mathrm{~mL})$ was stirred at room temperature for $4 \mathrm{~h}$. Solvent was removed, the residue was poured into $4 \mathrm{M} \mathrm{HCl}$ in dioxane $(8 \mathrm{~mL})$ and the mixture was stirred for $20 \mathrm{~h}$. Solvent was evaporated under vacuo, water $(2 \mathrm{~mL})$ was added and the resultant mixture was lyophilized to afford quantitatively tetraacid $25(170 \mathrm{mg})$ as a brown solid. ${ }^{1} \mathrm{H}$ NMR $\left(250 \mathrm{MHz}, \mathrm{D}_{2} \mathrm{O}\right) \delta=0.78(\mathrm{~s}, 3 \mathrm{H}, \mathrm{H} 5 / 6), 0.97(\mathrm{~s}, 3 \mathrm{H}$, $\mathrm{H} 5 / 6), 1.78$ (m, 1H, H4), 2.10 (m, 1H, H1/3/4), 2.30 (m, 2H, H1/3/4), 3.32 (m, 4H, H7), 3.98 (s, 4H, H8/16), 4.57 (s, 4H, H8/16), 7.62 (d, J = 7.6 Hz, 2H, H10), 8.00 (t, J = 7.7 Hz, 2H, H11), 8.09 (d, J = 7.7 Hz, 2H, H12) ppm; ${ }^{13} \mathrm{C}$ NMR (62.5 MHz, $\left.\mathrm{D}_{2} \mathrm{O}\right) \delta=14.8$ (C5,6); 27.4 (C4); 29.5 (C5,6); 36.7 (C1/3); 41.0 (C2); 54.1 (C7); 56.2 (C16); 27.8 (C8); 125.4 and 127.8 (C10/12); 139.8 (C11); 146.2 (C13); 149.1 (C9); 166.8 and 167.7 
(C14/17) ppm; IR v = 3363, 2360, 1730, 1630, $1400 \mathrm{~cm}^{-1}$; HRMS $\left(\mathrm{ESI}^{+}\right)$calcd m/z for $\mathrm{C}_{26} \mathrm{H}_{32} \mathrm{~N}_{4} \mathrm{O}_{8}[\mathrm{M}+$ $\mathrm{H}]^{+}$: 529.2293, found: 529.2283 .

\section{Conclusions}

In this work, we showed the suitability of several cyclobutane scaffolds for the preparation of different families of products with potential usefulness in the fields of surfactants, gelators and metal cation ligands. Enantiomerically pure cis-1,2-disubstitued cyclobutane derivatives have been efficient and selectively obtained from a common chiral half-ester. From this precursor, synthetic routes to amphiphiles as possible surfactants have been achieved through a cis- $\beta$-CBAA as a convenient intermediate. In addition, compounds with appropriate structures to behave as gelators have been synthesized from an orthogonally protected trans- $\beta$-CBAA. Otherwise, symmetric cis-1,3-cyclobutane scaffolds have been prepared from verbenone through norpinic acid that provided a cis-1,5-diamine, which is a key intermediate in the synthesis of different poly(amino acids). These compounds are valuable candidates to be investigated as polydentate ligands for lanthanide complexes. The properties of these families of compounds as well as their likely applications are the subject of active investigation in our laboratory.

Supplementary Materials: Supplementary materials can be found at http://www.mdpi.com/1422-0067/20/18/ 4333/s1.

Author Contributions: Conceptualization, O.I. and R.M.O.; Funding acquisition, O.I. and R.M.O.; Investigation, A.S., A.A., X.H. and G.C.; Methodology, O.I. and R.M.O.; Supervision, O.I. and R.M.O.; Writing-original draft, O.I. and R.M.O.; Writing - review \& editing, O.I. and R.M.O.

Funding: Financial support from Spanish Ministry of Economy and Competitivity (grant CTQ2016-77978-R, AEI/FEDER, UE).

Conflicts of Interest: There are no conflicts to declare.

\section{Abbreviations}

$\begin{array}{ll}\text { ATR } & \text { Attenuated Total Reflection } \\ \text { CBAA } & \text { Cyclobutane Amino Acid } \\ \text { DDS } & \text { Drug Delivery Systems } \\ \text { DIPEA } & \text { Diisopropylethylamine } \\ \text { DMAP } & \text { Dimethylaminopyridine } \\ \text { DMF } & \text { Dimethylformamide } \\ \text { DNA } & \text { Deoxyribonucleic Acid } \\ \text { ESI } & \text { Electrospray Ionization } \\ \text { HRMS } & \text { High-Resolution Mass Spectrometry } \\ \text { IR } & \text { Infrared } \\ \text { LMWG } & \text { Low Molecular-Weight Gelator } \\ \text { MS } & \text { Mass Spectrometry } \\ \text { NMR } & \text { Nuclear Magnetic Resonance } \\ \text { NPY } & \text { Neuropeptide Y } \\ \text { PyBOP } & \text { (Benzotriazol-1-yloxy)tripyrrolidinophosphonium hexafluorophosphate } \\ \text { QTOF } & \text { Quadrupole Time-of-Flight } \\ \text { TEA } & \text { Triethylamine } \\ \text { TFA } & \text { Trifluoroacetic Acid } \\ \text { THF } & \text { Tetrahydrofuran } \\ \text { TLC } & \text { Thin layer chromatography }\end{array}$

\section{References}

1. Sorrenti, A.; Illa, O.; Ortuño, R.M. Amphiphiles in aqueous solution: well beyond a soap bubble. Chem. Soc. Rev. 2013, 42, 8200-8219. [CrossRef] [PubMed] 
2. Dias, R.S.; Lindman, B. DNA Interaction with Polymers and Surfactants; Dias, R.S., Lindman, B., Eds.; John Wiley \& Sons, Inc.: Hoboken, NJ, USA, 2008.

3. Grueso, E.; Cerrillos, C.; Hidalgo, J.; Lopez-Cornejo, P. Compaction and Decompaction of DNA Induced by the Cationic Surfactant CTAB. Langmuir 2012, 28, 10968-10979. [CrossRef] [PubMed]

4. Foley, P.; Kermanshahi pour, A.; Beach, E.S.; Zimmerman, J.B. Derivation and Synthesis of Renewable Surfactants. Chem. Soc. Rev. 2012, 41, 1499-1518. [CrossRef] [PubMed]

5. Escuder, B.; Miravet, J.F. Functional Molecular Gels; Escuder, B., Miravet, J.F., Eds.; RSC Soft Matter Series Royal Society of Chemistry: Cambridge, UK, 2014.

6. Toth, S.É. Lacerda in Theranostics and Image Guided Drug Delivery; Thanou, M., Ed.; RSC: Cambridge, UK, 2018.

7. Merbach, A.; Helm, L.; Tóth, É. The Chemistry of Contrast Agents in Medical Magnetic Resonance Imaging, 2nd ed.; Merbach, A., Helm, L., Tóth, É., Eds.; Wiley: Weinheim, Germany, 2013.

8. Sorrenti, A.; Illa, O.; Pons, R.; Ortuño, R.M. Chiral Cyclobutane $\beta$-Amino Acid-Based Amphiphiles: Influence of cis/trans Stereochemistry on Solution Self-Aggregation and Recognition. Langmuir 2015, 31, 9608-9618. [CrossRef] [PubMed]

9. Sorrenti, A.; Illa, O.; Ortuño, R.M.; Pons, R. Chiral Cyclobutane $\beta$-Amino-Acid Based Amphiphiles: Influence of cis/trans Stereochemistry on Condensed Phase and Monolayer Structure. Langmuir 2016, 32, 6977-6984. [CrossRef] [PubMed]

10. Pi-Boleda, B.; Sorrenti, A.; Sans, M.; Illa, O.; Pons, R.; Branchadell, V.; Ortuño, R.M. Cyclobutane Scaffold in Bolaamphiphiles: Effect of Diastereoisomerism and Regiochemistry on Their Surface Activity Aggregate Structure. Langmuir 2018, 34, 11424-11432. [CrossRef] [PubMed]

11. Pi-Boleda, B.; Sans, M.; Campos, M.; Nolis, P.; Illa, O.; Estévez, J.C.; Branchadell, V.; Ortuño, R.M. Studies on cycloalkane-based organogelators: a new example of stochastic chiral symmetry-breaking induced by sonication. Chem. Eur. J. 2017, 23, 3357-3365. [CrossRef] [PubMed]

12. Pi-Boleda, B.; Campos, M.; Sans, M.; Basavilbaso, A.; Illa, O.; Branchadell, V.; Estévez, J.R.; Ortuño, R.M. Gaining insight into the influence of the hydroxyl groups on the gelling abilities of polyfunctional cyclohexane bisamides. Molecules 2019, 24, 352. [CrossRef] [PubMed]

13. Porcar-Tost, O.; Pi-Boleda, B.; García-Anton, J.; Illa, O.; Ortuño, R.M. Cyclobutane-based peptides/terpyridine conjugates: Their use in metal catalysis and as functional organogelators. Tetrahedron 2018, 74, 7252-7260. [CrossRef]

14. Izquierdo, S.; Rúa, F.; Sbai, A.; Parella, T.; Álvarez-Larena, A.; Branchadell, V.; Ortuño, R.M. (+)and (-)-2-Aminocyclobutane-1-carboxylic acids and their incorporation into highly rigid $\beta$-peptides: Stereoselective synthesis and a structural study. J. Org. Chem. 2005, 70, 7963-7971. [CrossRef] [PubMed]

15. Fernandes, C.; Gauzy, C.; Yang, Y.; Roy, O.; Pereira, E.; Faure, S.; Aitken, D.J. [2+2] Photocycloadditions with chiral uracil derivatives: access to all four stereoisomers of 2-aminocyclobutanecarboxylic acid. Synthesis 2007, 2222-2232. [CrossRef]

16. Torres, E.; Gorrea, E.; Da Silva, E.; Nolis, P.; Branchadell, V.; Ortuño, R.M. Prevalence of eight-membered hydrogen-bonded rings in some bis(cyclobutane) $\beta$-dipeptides with trans stereochemistry. Org. Lett. 2009, 11, 2301-2304. [CrossRef] [PubMed]

17. Torres, E.; Gorrea, E.; Burusco, K.K.; Da Silva, E.; Nolis, P.; Rúa, F.; Boussert, S.; Díez-Pérez, I.; Dannenberg, S.; Izquierdo, S.; et al. Folding and self-assembling with $\beta$-oligomers based on $(1 R, 2 S)$-2-aminocyclobutane1-carboxylic acid. Org. Biomol. Chem. 2010, 8, 564-575. [CrossRef] [PubMed]

18. Gorrea, E.; Pohl, G.; Nolis, P.; Celis, S.; Burusco, K.K.; Branchadell, V.; Perczel, A.; Ortuño, R.M. Secondary structure of short $\beta$-peptides as the chiral expression of monomeric building units: A rational and predictive model. J. Org. Chem. 2012, 77, 9795-9806. [CrossRef] [PubMed]

19. Gorrea, E.; Torres, E.; Nolis, P.; da Silva, E.; Amabilino, D.B.; Branchadell, V.; Ortuño, R.M. Self-assembly of chiral trans-cyclobutane containing $\beta$-dipeptides into ordered aggregates. Chem. Eur. J. 2011, 17, 4588-4597. [CrossRef] [PubMed]

20. Celis, S.; Nolis, P.; Branchadell, V.; Illa, O.; Ortuño, R.M. Low-molecular-weight gelators consisting of hybrid cyclobutane-based peptides. Org. Biomol. Chem. 2013, 11, 2839-2846. [CrossRef] [PubMed]

21. Berlicki, Ł.; Kaske, M.; Gutiérrez-Abad, R.; Bernhardt, G.; Illa, O.; Ortuño, R.M.; Cabrele, C.; Buschauer, A.; Reiser, O. Replacement of $\mathrm{Th}^{32}$ and $\mathrm{Gln}^{34}$ in the C-terminal neuropeptide $\mathrm{Y}$ fragment 25-36 by cis-cyclobutaneand cis-cyclopentane $\beta$-amino acids shifts selectivity toward the $\mathrm{Y}_{4}$ receptor. J. Med. Chem. 2013, 56, 8422-8431. [CrossRef] [PubMed] 
22. Mayans, E.; Gargallo, A.; Illa, O.; Ortuño, R.M. Diastereodivergent synthesis of chiral cyclobutane scaffolds: 1,3-amino alcohols, 1,3-diamines and some derivatives. Eur. J. Org. Chem. 2013, 2013, 1425-1433. [CrossRef]

23. Sans, M.; Illa, O.; Ortuño, R.M. Stereoselective synthesis of all stereoisomers of orthogonally protected cyclobutane-1,2-diamine and some chemoselective transformations. Org. Lett. 2012, 14, 2431-2433. [CrossRef]

24. Andre, V.; Gras, M.; Awada, H.; Guillot, R.; Robin, S.; Aitken, D.J. A unified synthesis of all stereoisomers of (2-aminomethyl)cyclobutane-1-carboxylic acid. Tetrahedron 2013, 69, 3571-3576. [CrossRef]

25. Awada, H.; Robin, S.; Guillot, R.; Yazbeck, O.; Naoufal, D.; Jaber, N.; Hachem, A.; Aitken, D.J. Practical syntheses of both enantiomers of the conformationally restricted GABA analogue cis-(2-aminocyclobutyl)acetic acid. Eur. J. Org. Chem. 2014, 2014, 7148-7155. [CrossRef]

26. Ji, X.; Huang, H. Synthetic methods for 1,3-diamines. Org. Biomol. Chem. 2016, 14, 10557-10566. [CrossRef] [PubMed]

27. Illa, O.; Porcar-Tost, O.; Robledillo, C.; Elvira, C.; Nolis, P.; Branchadell, V.; Reiser, O.; Ortuño, R.M. Stereoselectivity of Proline/Cyclobutane Amino Acid-Containing Peptide Organocatalysts for Asymmetric Aldol Additions: A Rationale. J. Org. Chem. 2018, 83, 350-363. [CrossRef]

28. Gutiérrez-Abad, R.; Illa, O.; Ortuño, R.M. Synthesis of chiral cyclobutane containing $C_{3}$-symmetric peptide dendrimers. Org. Lett. 2010, 12, 3148-3151. [CrossRef]

29. Gorrea, E.; Carbajo, D.; Gutiérrez-Abad, R.; Illa, O.; Royo, M.; Ortuño, R.M. Searching for new cell-penetrating agents: hybrid cyclobutane-proline $\gamma, \gamma$-peptides. Org. Biomol. Chem. 2012, 10, 4050-4057. [CrossRef]

30. Kiss, L.; Fülöp, F. Synthesis of carbocyclic and heterocyclic $\beta$-aminocarboxylic acids. Chem. Rev. 2014, 114, 1116-1169. [CrossRef] [PubMed]

31. Park, J.D.; Allphin, N.L., Jr.; Choi, S.K.; Settine, R.L.; Hedrick, G.W. Hydroxyalky and Olefinic Substituted gem-Dimethylcyclobutanes. Ind. Eng. Chem. Prod. Res. Dev. 1965, 4, 149-153. [CrossRef]

32. Forgács, A.; Giovenzana, G.B.; Botta, M.; Brücher, E.; Tóth, I.; Baranyai, Z. Influence of gem-dimethyl substitution on the stability, kinetics and relaxometric properties of PDTA complexes. Eur. J. Inorg. Chem. 2012, 2012, 2074-2086. [CrossRef]

33. Gale, E.M.; Kenton, N.; Caravan, P. $\left[\mathrm{Gd}(\mathrm{CyPic} 3 \mathrm{~A})\left(\left(\mathrm{H}_{2} \mathrm{O}\right)_{2}\right]^{-}\right.$: A stable bis(aquated) and high-relaxivity Gd(III) complex. Chem. Commun. 2013, 49, 8060-8062. [CrossRef]

34. Tircsó, G.; Regueiro-Figueroa, M.; Nagy, V.; Garda, Z.; Garai, T.; Kálmán, F.K.; Esteban-Gómez, D.; Tóth, É.; Platas-Iglesias, C. Approaching the kinetic inertness of macrocyclic gadolinium(III)-based MRI contrast agents with highly rigid open-chain derivatives. Chem. Eur. J. 2016, 22, 896-901. [CrossRef]

35. Thiele, N.A.; Brown, V.; Kelly, J.M.; Amor-Coarasa, A.; Jermilova, U.; MacMillan, S.N.; Nikolopoulou, A.; Ponnala, S.; Ramogida, C.F.; Robertson, A.K.H.; et al. An eighteen-membered macrocyclic ligand for actinium-225 targeted alpha therapy. Angew. Chem. Int. Ed. 2017, 56, 14712-14717. [CrossRef] [PubMed]

36. Thiele, N.A.; MacMillan, S.N.; Wilson, J.J. Rapid dissolution of $\mathrm{BaSO}_{4}$ by macropa, an 18-membered macrocycle with high affinity for $\mathrm{Ba}^{2+}$. J. Am. Chem. Soc. 2018, 140, 17071-17078. [CrossRef] [PubMed]

37. Kálmán, F.K.; Végh, A.; Regueiro-Figueroa, M.; Tóth, É.; Platas-Iglesias, C.; Tircsó, G. H4 octapa: highly stable complexation of lanthanide(III) ions and copper(II). Inorg. Chem. 2015, 54, 2345-2356. [CrossRef] [PubMed] 